\title{
Potential drug interaction between paclitaxel and clopidogrel
}

\author{
YASUTAKA SHINODA, MICHIO KIMURA, EISEKI USAMI, HIROKI ASANO and TOMOAKI YOSHIMURA
}

\author{
Department of Pharmacy, Ogaki Municipal Hospital, Ogaki, Gifu 503-8502, Japan
}

Received February 18, 2016; Accepted May 11, 2016

DOI: $10.3892 /$ br.2016.685

\begin{abstract}
Paclitaxel is mainly inactivated in vivo by cytochrome P5402C8 (CYP2C8). In recent years, the clopidogrel metabolite has been reported to potently inhibit CYP2C8. However, clinical information regarding the interaction between these two drugs is limited. To the best of our knowledge, this is the first retrospective study investigating the potential for the drug interaction between paclitaxel and clopidogrel. A total of 8 cases in which clopidogrel and paclitaxel were used in combination were examined. The incidence of adverse events and discontinuation rate in these cases were assessed. Neutrophil counts were compared in patients prior and subsequent to the combined administration of clopidogrel and paclitaxel. Grade 3 neutropenia occurred in all cases of combination therapy and grade 4 occurred in 7 cases $(88 \%)$. In addition, 4 cases $(50 \%)$ showed febrile neutropenia. Four cases (50\%) involved a severe adverse event requiring discontinuation of drug administration. In 1 case involving 6 courses of paclitaxel and nedaplatin therapy prior and subsequent to clopidogrel, there was a significant reduction in the average neutrophil count after 8 days of combination treatment $\left(1,240 \pm 395\right.$ counts $/ \mathrm{mm}^{3}$ without clopidogrel; $370 \pm 148$ counts $/ \mathrm{mm}^{3}$ with clopidogrel; mean \pm standard deviation, $\mathrm{P}<0.01$ ). Drug interactions during co-administration of clopidogrel and paclitaxel may cause severe neutropenia. To avoid these interactions, alternative medications should be considered. If these two drugs are used in combination, it may be necessary to monitor for adverse events more carefully.
\end{abstract}

\section{Introduction}

In recent years, it has become increasingly common for cancer chemotherapy to be administered even to patients with underlying diseases. However, there is limited clinical data for

Correspondence to: Mr. Yasutaka Shinoda, Department of Pharmacy, Ogaki Municipal Hospital, 4-86 Minaminokawa-cho, Ogaki, Gifu 503-8502, Japan

E-mail: shonoda065039@gmail.com

Key words: clopidogrel, paclitaxel, drug interaction, CYP2C8, cytochrome $\mathrm{P} 450$ drug-drug interactions between anticancer agents and other disease therapeutics.

Paclitaxel has been widely used as a key drug for a number of cancers including ovarian cancer and lung cancer. The major metabolic pathway of paclitaxel is via cytochrome P5402C8 (CYP2C8), which deactivates it to $6 \alpha$-hydroxide (1). Clopidogrel is a common antiplatelet agent used to inhibit blood clots. Clopidogrel is metabolized in the liver to acyl- $\beta$-D-glucuronide, which has been recently shown to inhibit CYP2C8 (2). Therefore, the potential exists for paclitaxel and clopidogrel to cause drug interactions, but the combination has rarely been examined clinically (3).

In the present study, patients who had been administered the combination of paclitaxel and clopidogrel were investigated. To the best of our knowledge, this is the first retrospective study investigating the potential interaction between these two drugs.

\section{Materials and methods}

Background. Patients who were administered a combination of paclitaxel and clopidogrel (75 mg/day) at Ogaki Municipal Hospital (Ogaki, Gifu, Japan) between January 2009 and October 2015 were identified. The gender, age, cancer type, regimen, paclitaxel dose, kidney function, liver function and any adverse events were extracted from the electronic medical record. The severity of adverse events was evaluated using the Common Terminology Criteria for Adverse Events (version 4.0; http://evs.nci.nih.gov/ftp1/CTCAE/About.html). The present study was conducted with the approval of the Ogaki Municipal Hospital ethics committee.

Adverse events and discontinuation rate. The incidence of adverse events was analyzed, including the discontinuation rate due to adverse events. These rates were compared with typical clinical trials $(4,5)$.

Comparison of average neutrophil count prior and subsequent to administration of clopidogrel and paclitaxel. Neutrophil counts and neutrophil reduction rate were recorded and compared in patients prior and subsequent to the combined administration of clopidogrel and paclitaxel. Neutrophil reduction rate was calculated by this formula: [1 - (day 8 neutrophil counts/day 1 neutrophil counts)] x $100(\%)$.

Statistical analysis. Average neutrophil counts prior and subsequent to co-administration of clopidogrel and paclitaxel were 


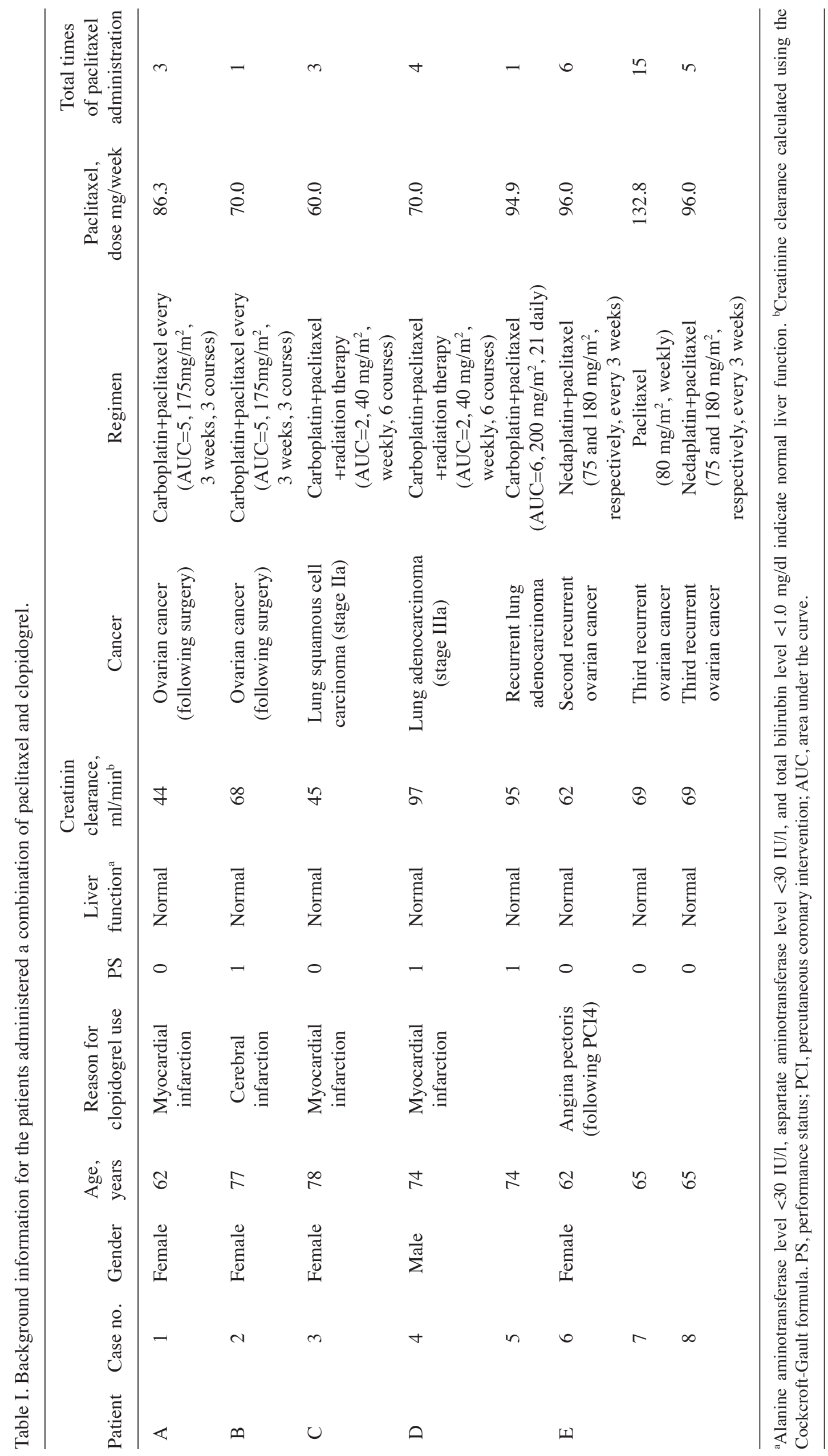


Table II. Summary of the adverse events (AE).

\begin{tabular}{|c|c|c|c|c|c|c|c|c|}
\hline \multirow[b]{2}{*}{ Case no. } & \multirow{2}{*}{$\begin{array}{l}\text { Received } \\
\text { courses, } \mathrm{n}\end{array}$} & \multirow{2}{*}{$\begin{array}{l}\text { Dose reduction } \\
\text { or skip }\end{array}$} & \multicolumn{2}{|c|}{$\begin{array}{l}\text { Average cell } \\
\text { count after } 8 \text { days } \\
\text { of paclitaxel, } \\
\text { administration } \\
\text { counts } / \mathrm{mm}^{3}\end{array}$} & \multicolumn{2}{|c|}{$\begin{array}{l}\text { Minimum cell count } \\
\text { in all courses, } \\
\text { counts } / \mathrm{mm}^{3}\end{array}$} & \multirow{2}{*}{$\begin{array}{c}\text { Febrile } \\
\text { neutropenia }\end{array}$} & \multirow{2}{*}{$\begin{array}{c}\text { Administration } \\
\text { end reason }\end{array}$} \\
\hline & & & Leucocyte & Neutrophil & Leucocyte & Neutrophil & & \\
\hline 1 & 3 & Yes & 1,243 & 140 & 960 & 100 & Yes & Protocol finished \\
\hline 2 & 1 & No & - & - & 860 & 340 & Yes & Discontinued for $\mathrm{AE}$ \\
\hline 3 & 1 & Yes & 2,097 & 1,047 & 1,350 & 710 & No & Discontinued for $\mathrm{AE}$ \\
\hline 4 & 1 & Yes & 1,655 & 1,040 & 620 & 300 & Yes & Protocol finished \\
\hline 5 & 1 & Yes & - & - & 640 & 190 & Yes & Discontinued for $\mathrm{AE}$ \\
\hline 6 & 6 & No & 1,148 & 370 & 840 & 230 & No & Completely response \\
\hline 7 & 7 & Yes & 1,781 & 875 & 850 & 250 & No & Progressive disease \\
\hline 8 & 9 & Yes & 818 & 200 & 410 & 50 & No & Discontinued for $\mathrm{AE}$ \\
\hline
\end{tabular}
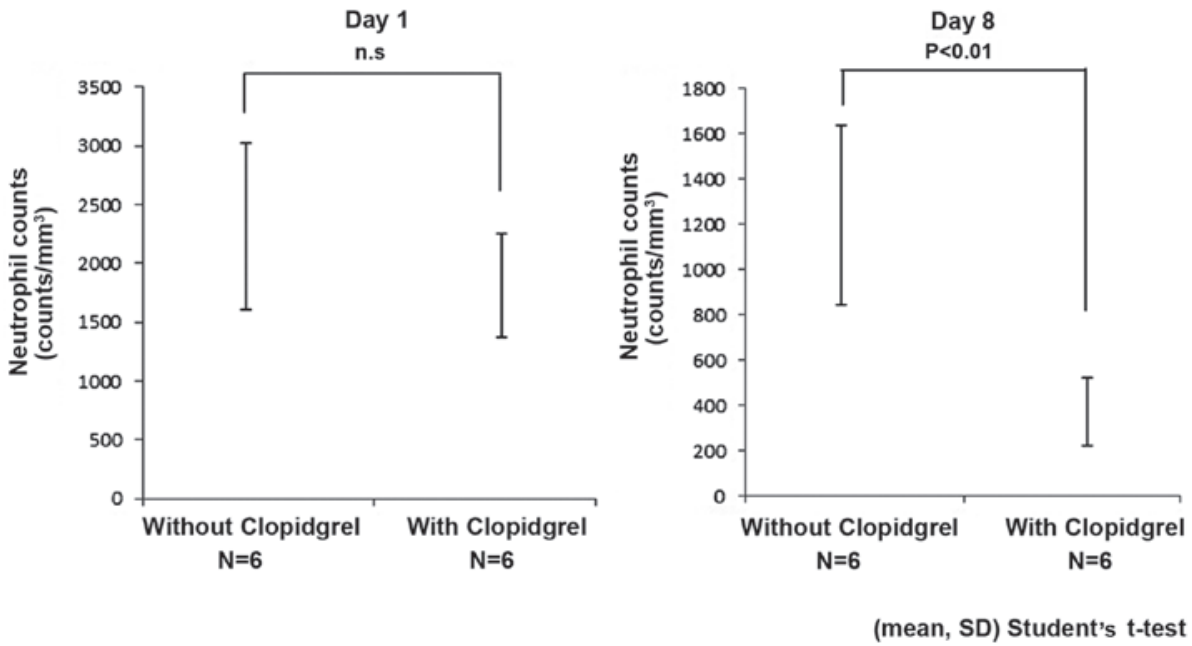

Figure 1. Comparison of neutrophil counts prior and subsequent to administration of clopidogrel (mean \pm SD). SD, standard deviation; n.s., not significant.

compared using an unpaired t-test, and the neutrophil reduction rate was compared using Mann-Whitney U-test with $\mathrm{P}<0.05$ considered to indicate a statistically significant difference. The statistical analysis software used was EZR version 1.26 (6).

\section{Results}

Background. Patient characteristics and background are shown in Table I. A total of 5 patients received paclitaxel and clopidogrel concomitantly. The therapeutic regimen for the patients included was carboplatin (nedaplatin) + paclitaxel (4 cases), paclitaxel alone (1 case), carboplatin + paclitaxel + radiation therapy ( 2 cases), or carboplatin + paclitaxel (1 case). A total of 8 cases were analyzed. The only drug used that influences CYP2C8 was clopidogrel. None of the 8 cases had any notable problems regarding blood cell counts prior to chemotherapy.

Adverse events and discontinuation rate. A summary of the adverse events for each case is shown in Table II. Grade 3 $\left(<1,000\right.$ to 500 counts $\left./ \mathrm{mm}^{3}\right)$ or higher neutropenia presented in all cases, occurring from the first course of treatment in 5 cases. Grade 4 neutropenia $\left(<500\right.$ counts $/ \mathrm{mm}^{3}$ ) was observed in 5 cases $(88 \%)$ and 4 cases showed febrile neutropenia (50\%). Treatment was discontinued due to adverse events in 4 cases (50\%). Reduction of paclitaxel dose or skipping a course of treatment was required in 6 cases $(75 \%)$.

Comparison of average neutrophil counts prior and subsequent to combination of clopidogrel and paclitaxel. The data include the findings for 1 patient who underwent 6 courses of paclitaxel + nedaplatin therapy for a second recurrent ovarian cancer and 3 years later had 6 courses of paclitaxel + nedaplatin therapy for a third recurrence. Data were not available on potential bone marrow suppressive therapy between courses of chemotherapy.

On the initial day of paclitaxel administration (day 1), average neutrophil counts showed no significant difference with or without clopidogrel administration (without clopidogrel, $2,312 \pm 712$ counts $/ \mathrm{mm}^{3}$; with clopidogrel, $1,815 \pm 438$ counts $/ \mathrm{mm}^{3}$, 
mean \pm standard deviation; $\mathrm{P}=0.18)$. At day 8 of paclitaxel administration, the average neutrophil counts with clopidogrel co-administration were significantly reduced (withoutclopidogrel, 1,240 \pm 395 counts $/ \mathrm{mm}^{3}$; with clopidogrel, 370 \pm 148 counts $/ \mathrm{mm}^{3}$, mean \pm standard deviation; $\mathrm{P}<0.01$ ) (Fig. 1 ).

The neutrophil reduction rate was significantly higher in combination with clopidogrel compared to without clopidogrel [without clopidogrel: $54.1 \%$ (39.5-59.9\%); with clopidogrel: $82.6 \%$ (60.5-89.4\%), median (range); $\mathrm{P}<0.01]$.

\section{Discussion}

Paclitaxel is believed to be mainly inactivated to $6 \alpha$-hydroxide by CYP2C8 (1). Poor metabolism by CYP2C8 reduces the clearance of paclitaxel by $11 \%$. An increased neuropathy risk by 2-3 times has been reported (7-9). In addition, this has been shown to increase the risk of leucopenia $(10,11)$. Therefore, drug interaction with CYP2C8 inhibitors is assumed to increase the incidence and severity of adverse events of paclitaxel.

Clopidogrel is metabolized in the liver and the active metabolite has been shown to inhibit CYP2C8 (2). It has been reported that the activity of CYP2C8 is inhibited $60-85 \%$ by continuous administration of $75 \mathrm{mg}$ clopidogrel/day; thus, its inhibitory effect is shown to be strong. When clopidogrel is used in combination with a drug metabolized by CYP2C8, it may decrease the drug clearance. The interaction of paclitaxel and clopidogrel was previously reported by Bergmann et al (3) in a case study; the clearance of paclitaxel was reduced $38 \%$ by the co-administration of clopidogrel. Despite this warning of the potential drug interactions of the two agents, follow-up clinical studies are lacking.

The variation in the background of the cases in this study makes a simple comparison difficult. Paclitaxel + carboplatin therapy (TC therapy) was used as the standard regimen for treatment of ovarian cancer and lung cancer. TC therapy is associated with a relatively high rate of neutropenia compared to other paclitaxel regimens (grade 3 or 4 leukopenia: $59 \%$, grade 3 or 4 neutropenia: $89-92 \%$, febrile neutropenia: $9 \%$ ) $(4,5)$. However, TC therapy administration for $>6$ courses has reported a rate of $87 \%$ neutropenia and is well tolerated (4). Although comparisons between different regimens are difficult, in previous studies, patients received more carboplatin (AUC 6 and 7.5) compared to the patients in the present study. The paclitaxel doses were similar to previous studies $\left(175\right.$ and $\left.180 \mathrm{mg} / \mathrm{m}^{2}\right)$ and the majority of patients in the present study. Therefore, the neutropenia risk is considered lower compared to these studies. However, in the present study, neutropenia of grade 3 or higher presented in all cases, and $50 \%$ discontinued treatment with severe adverse events such as febrile neutropenia. This suggests that the adverse events are amplified by the drug interactions of paclitaxel and clopidogrel. A larger study that can control for patient background is required in order to further quantify this drug interaction.

For the 1 case involving paclitaxel + nedaplatin therapy, it was possible to compare the average neutrophil counts prior and subsequent to clopidogrel administration. The case also used aspirin, atorvastatin and lansoplazole, following percutaneous coronary intervention. Except for clopidogrel, these drugs cannot be considered to influence drug interaction with paclitaxel, and bone marrow suppression. The neutrophil reduction rate was significantly higher following the combination treatment of clopidogrel and paclitaxel compared to prior to clopidogrel administration. Infection did not occur in this case, but the average number of neutrophils at day 8 was $<500$ counts $/ \mathrm{mm}^{3}$ with clopidogrel. In general, infection rates increase when neutrophil counts fall $<500$ counts $/ \mathrm{mm}^{3}$, and the frequency and severity of infections are inversely proportional to the number of neutrophils (12). Thus, when neutropenia is severe due to the administration of clopidogrel, it is likely that the risk of infection is also greatly increased.

The present study has certain limitations. One of them is the small sample size ( 8 cases). Patient backgrounds were not matched in each case, due to the different regimens. Additionally, only 1 patient could be evaluated who received paclitaxel with and without clopidogrel. Therefore, the impact of aging is evident in prior and subsequent comparison of a single case. Furthermore, the study was not a pharmacogenetic and pharmacokinetic study. Therefore, more studies are required.

The drug interaction of paclitaxel and clopidogrel cannot be clinically negligible, as the data suggest that there is an increased risk of severe adverse events. Therefore, therapeutic strategies should be considered to avoid the combination of these two agents where possible. When a combination is required, it is necessary to monitor for adverse events carefully.

\section{References}

1. Rahman A, Korzekwa KR, Grogan J, Gonzalez FJ and Harris JW: Selective biotransformation of taxol to 6 alpha-hydroxytaxol by human cytochrome P450 2C8. Cancer Res 54: 5543-5546, 1994.

2. Tornio A, Filppula AM, Kailari O, Neuvonen M, Nyrönen TH, Tapaninen T, Neuvonen PJ, Niemi $\mathrm{M}$ and Backman JT: Glucuronidation converts clopidogrel to a strong time-dependent inhibitor of CYP2C8: A phase II metabolite as a perpetrator of drug-drug interactions. Clin Pharmacol Ther 96: 498-507, 2014.

3. Bergmann TK, Filppula AM, Launiainen T, Nielsen F, Backman J and Brosen K: Neurotoxicity and low paclitaxel clearance associated with concomitant clopidogrel therapy in a 60 year old Caucasian woman with ovarian carcinoma. Br J Clin Pharmacol: Oct 7, 2015 (Epub ahead of print).

4. Ozols RF, Bundy BN, Greer BE, Fowler JM, Clarke-Pearson D, Burger RA, Mannel RS, DeGeest K, Hartenbach EM and Baergen R; Gynecologic Oncology Group: Phase III trial of carboplatin and paclitaxel compared with cisplatin and paclitaxel in patients with optimally resected stage III ovarian cancer: A Gynecologic Oncology Group study. J Clin Oncol 21: 3194-3200, 2003.

5. Katsumata N, Yasuda M, Takahashi F, Isonishi S, Jobo T, Aoki D, Tsuda H, Sugiyama T, Kodama S, Kimura E, et al; Japanese Gynecologic Oncology Group: Dose-dense paclitaxel once a week in combination with carboplatin every 3 weeks for advanced ovarian cancer: A phase 3, open-label, randomised controlled trial. Lancet 374: 1331-1338, 2009.

6. Kanda Y: Investigation of the freely available easy-to-use software 'EZR' for medical statistics. Bone Marrow Transplant 48: 452-458, 2013.

7. Bergmann TK, Brasch-Andersen C, Gréen H, Mirza M, Pedersen RS, Nielsen F, Skougaard K, Wihl J, Keldsen N, Damkier P, et al: Impact of CYP2C8*3 on paclitaxel clearance: A population pharmacokinetic and pharmacogenomic study in 93 patients with ovarian cancer. Pharmacogenomics J 11: 113-120, 2011.

8. Hertz DL, Roy S, Motsinger-Reif AA, Drobish A, Clark LS, McLeod HL, Carey LA and Dees EC: CYP2C8*3 increases risk of neuropathy in breast cancer patients treated with paclitaxel. Ann Oncol 24: 1472-1478, 2013. 
9. Hertz DL, Motsinger-Reif AA, Drobish A, Winham SJ, McLeod HL, Carey LA and Dees EC: CYP2C $8 * 3$ predicts benefit/risk profile in breast cancer patients receiving neoadjuvant paclitaxel. Breast Cancer Res Treat 134: 401-410, 2012.

10. Huizing MT, Vermorken JB, Rosing H, ten Bokkel Huinink WW, Mandjes I, Pinedo HM and Beijnen JH: Pharmacokinetics of paclitaxel and three major metabolites in patients with advanced breast carcinoma refractory to anthracycline therapy treated with a 3-hour paclitaxel infusion: A European Cancer Centre (ECC) trial. Ann Oncol 6: 699-704, 1995.
11. Nakajima M, Fujiki Y, Kyo S, Kanaya T, Nakamura M, Maida Y, Tanaka M, Inoue M and Yokoi T: Pharmacokinetics of paclitaxel in ovarian cancer patients and genetic polymorphisms of CYP2C8, CYP3A4, and MDR1. J Clin Pharmacol 45: 674-682, 2005.

12. National Comprehensive Cancer Network: Prevention and treatment of cancer-related infections. Version 1.2013. http://oralcancerfoundation.org/treatment/pdf/infections.pdf. Accessed February 5, 2013. 